PROCEEDINGS OF THE

AMERICAN MATHEMATICAL SOCIETY

Volume 132, Number 11, Pages 3411-3415

S 0002-9939(04)07515-X

Article electronically published on June 16, 2004

\title{
CYCLE DECOMPOSITIONS AND TRAIN TRACKS
}

\author{
CHARLES A. MATTHEWS AND DAVID J. WRIGHT \\ (Communicated by Alan Dow)
}

\begin{abstract}
We prove that the disjoint cycle decomposition of the permutation $\left(12 \cdots n_{1}\right)^{k_{1}}\left(12 \cdots n_{2}\right)^{k_{2}} \cdots\left(12 \cdots n_{r}\right)^{k_{r}}$ consists of cycles of at most $r$ distinct lengths. The proof relies on the geometry and topology of simple closed curves and train tracks on a closed surface of genus $r$.
\end{abstract}

It is easy to see that the disjoint cycle decomposition of the permutation $(12 \cdots n)^{k}$ consists of cycles that all have the same length; the number of disjoint cycles is the greatest common divisor of $n$ and $k$.

We prove the following generalization of this result:

Theorem. Let $n_{i}$ and $k_{i}$ be positive integers for $1 \leq i \leq r$. Then the disjoint cycle decomposition of the permutation $\rho=\left(12 \cdots n_{1}\right)^{\bar{k}_{1}} \cdots\left(12 \cdots n_{r}\right)^{k_{r}}$ consists of cycles of at most $r$ distinct lengths.

Although the theorem seems like a very basic and elementary result about permutations and is purely algebraic in nature, we know of no purely algebraic or elementary proof. We supply a proof that uses topology, making the theorem a good example of how topology can be used to prove a new result in algebra. Our proof consists of producing a multiple curve on a smooth surface of genus $r$ which represents the permutation $\rho$ in such a way that disjoint cycles in the disjoint cycle decomposition of $\rho$ correspond to connected components of the multiple curve. Cycles of distinct lengths will correspond to nonhomotopic components. We then show that the multiple curve can have at most $r$ nonhomotopic components by producing a train track on the surface that carries the multiple curve and analyzing the train track. It follows that the cycles of $\rho$ can have at most $r$ distinct lengths.

Train tracks were developed by Thurston in [5] and are discussed in detail in [4]. Our results arose in the context of the problem of determining the number of components of a multiple curve specified by integral weights on a train track. (This problem is discussed for the closed surface of genus 2 in [3.)

Start by conjugating $\rho$ so that $n_{1}$ is the largest of the integers $n_{i}, 1 \leq i \leq r$. (It is a well-known fact that two permutations are conjugate if and only if they have the same number of cycles of each length; see 2] or [1, for example.) Next, since $\left(12 \cdots n_{i}\right)^{k_{i}}=\left(12 \cdots n_{i}\right)^{k_{i} \bmod n_{i}}$, assume $0<k_{i}<n_{i}$ for each $i$. In the product of permutations, we use the convention of applying the permutation on the right first. (Some authors prefer to apply the permutation on the left first.) For example,

Received by the editors February 18, 2002 and, in revised form, November 10, 2002.

2000 Mathematics Subject Classification. Primary 57N99, 20B30, 32G15, 30F99.

Key words and phrases. Cycle decomposition, train track, multiple curve.

(C)2004 American Mathematical Society 


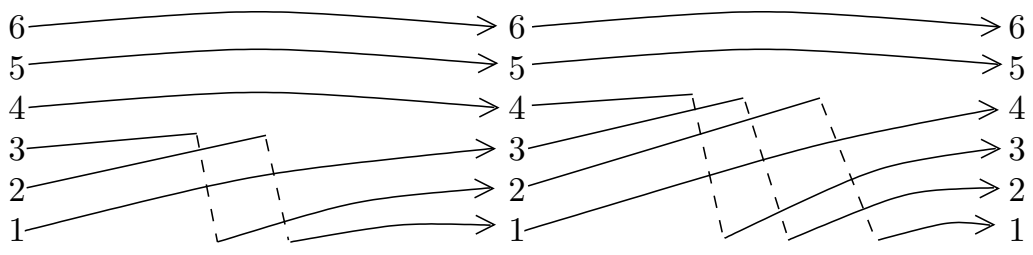

Figure 1. The first two factors $(123)^{2}$ and $(1234)^{3}$ in the permutation $(123456)^{4}(1234)^{3}(123)^{2}$ acting on the integers from 1 to 6 .

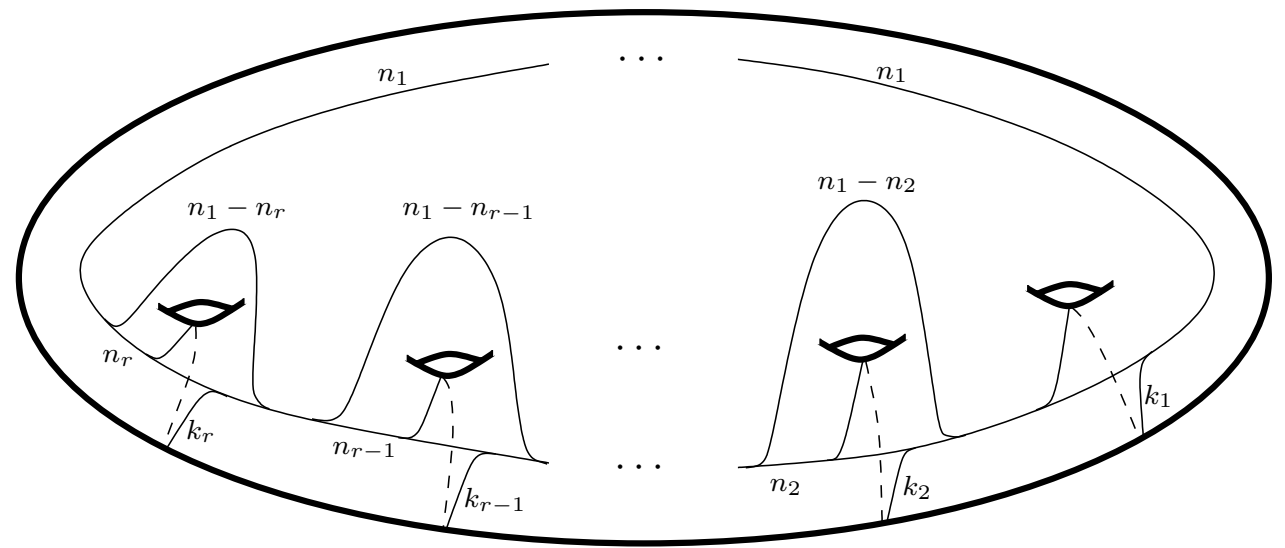

Figure 2. A train track $\tau$ on a closed surface of genus $r$. The surface appears in boldface; $\tau$ does not. The multiple curve $C$ that embodies the permutation $\rho=\left(12 \cdots n_{1}\right)^{k_{1}} \cdots\left(12 \cdots n_{r}\right)^{k_{r}}$ is specified by the integral weights shown on the branches of $\tau$.

$(1234)(24)=(12)(34)$ but $(24)(1234)=(14)(23)$. So the first permutation in the product $\rho=\left(12 \cdots n_{1}\right)^{k_{1}} \cdots\left(12 \cdots n_{r}\right)^{k_{r}}$ fixes the integers from $n_{r}+1$ to $n_{1}$, and shifts the integers 1 through $n_{r}$ by adding $k_{r}$ (modulo $n_{r}$ ) as in Figure 1 .

Figure 1 suggests that we might study the combinatorics of multiple curves on surfaces to understand the cycle decomposition of $\rho$. Let $S_{r}$ denote a closed, smooth surface of genus $r$. A multiple curve on $S_{r}$ is a collection of disjoint, smooth, simple closed curves on $S_{r}$, none of which is homotopically trivial. The multiple curve $C$ that we construct, which embodies the permutation $\rho$, is carried by the train track $\tau$ shown in Figure 2. (For a definition and discussions of train tracks, see 4]. Essentially, a train track carries a multiple curve if the curves can be squashed together smoothly so that they are contained in the train track. The integers labeling the branches of the train track in Figure 2 represent the numbers of curve arcs from $C$ squashed together on that branch.)

Label the curve arcs carried in the branch of $\tau$ near the top of Figure 2 with the integers from 1 to $n_{1}$. Figure 3 shows this enumeration for the permutation $\rho=(12345)^{3}(123)^{2}$ on a surface of genus 2 . For $1 \leq j \leq n_{1}$, the permutation $\rho$ takes $j$ to the label of the next arc traversed as one would travel in a counterclockwise direction along the multiple curve $C$ carried by $\tau$. Thus, disjoint cycles in the disjoint cycle decomposition of $\rho$ correspond to connected components of 


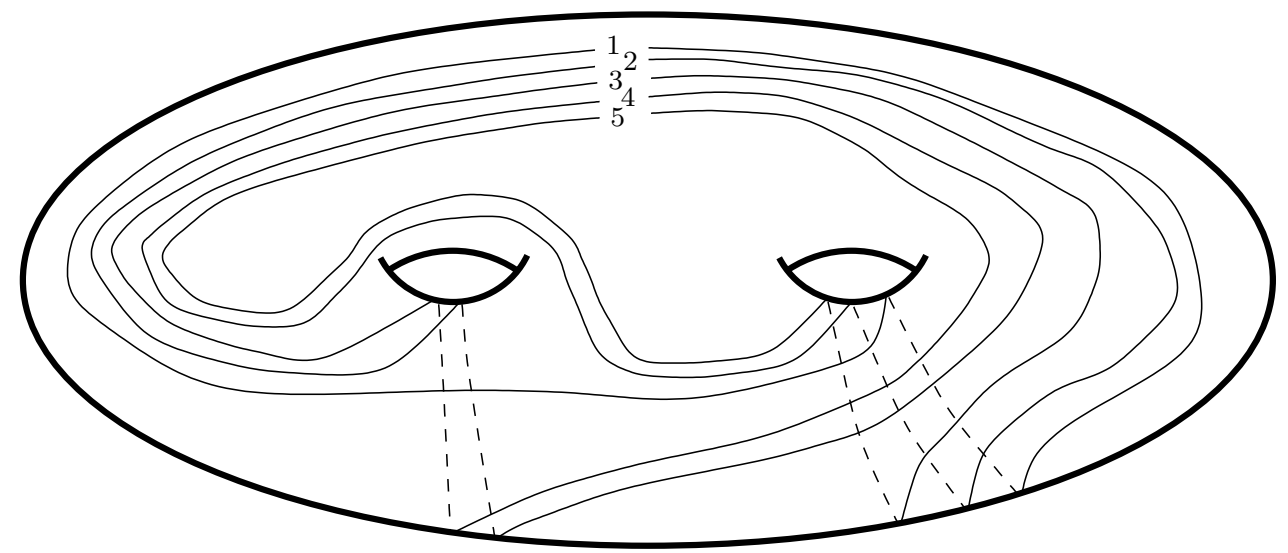

Figure 3 . The multiple curve $C$ that represents the permutation $\rho=(12345)^{3}(123)^{2}$.

$C$, and disjoint cycles of distinct lengths correspond to nonhomotopic components. (Disjoint cycles of the same length correspond to components of $C$ that may or may not be homotopic.) If we show that $C$ has at most $r$ nonhomotopic components, this will finish the proof of our theorem.

It is not difficult to see that one can smoothly deform the curves in $C$ (or deform $\tau$ ) so that all the switches of $\tau$ lie in a single component $X$ of $S_{r}-C$ (see Figure (4).

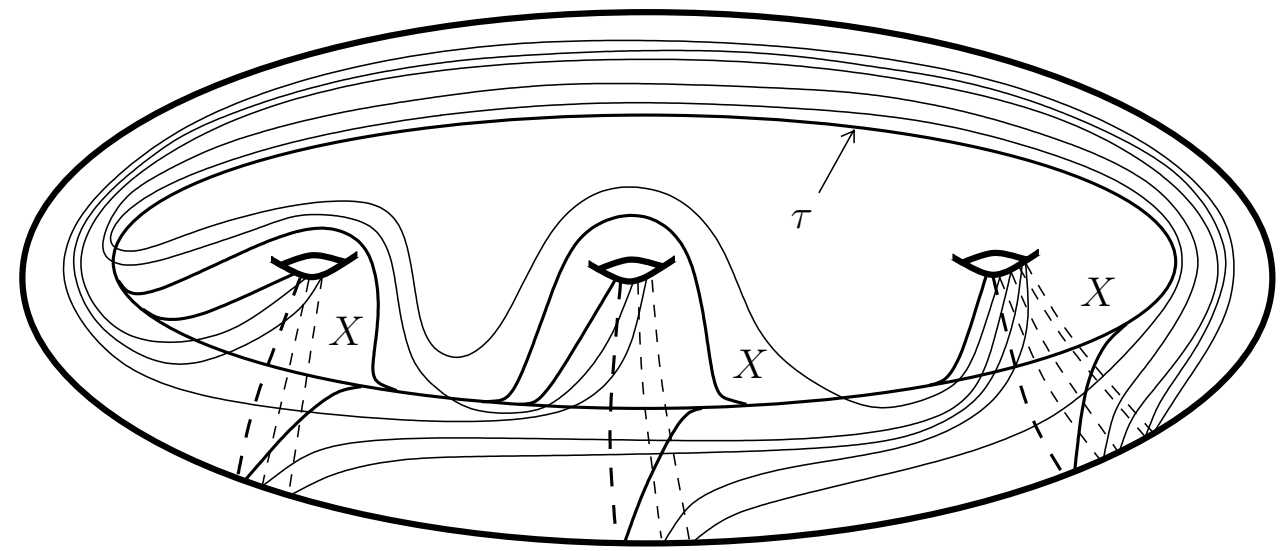

Figure 4. The surface $S_{3}$ along with the train track $\tau$ and a multiple curve $C$ carried by $\tau$. We have arranged the curves in $C$ so that all the switches of $\tau$ are in a single component $X$ of $S_{3}-C$. The switches are located between the arcs of curves in $C$ that pass through the handles and those that avoid the handles from above.

Cutting the surface $S_{r}$ along $\tau$ produces a connected polygon with $4 r-2$ cuspidal vertices arising from the $4 r-2$ switches on $\tau$. (Each of the $r$ handles of $S_{r}$ is removed by cutting along $\tau$, and $S_{r}-\tau$ is connected. We will show momentarily that $S_{r}-\tau$ 
is simply connected.) The component $X$ of $S_{r}-C$ has a smooth boundary. So in order to obtain this component from the polygon $S_{r}-\tau$, all of the cuspidal vertices must be identified in pairs, as in Figure 5 .

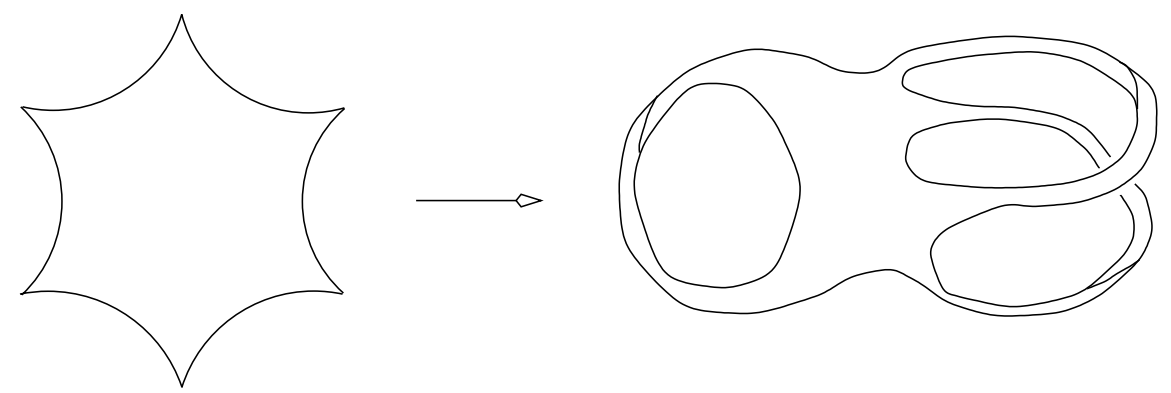

Figure 5. One way to identify the cuspidal vertices of the polygon $S_{2}-\tau$ in pairs, making the boundary smooth.

The polygon $S_{r}-\tau$ has Euler characteristic at most 1 (allowing for the possibility that $S_{r}-\tau$ is not simply connected), and each identification of a pair of cuspidal vertices reduces the Euler characteristic by 1 . So the Euler characteristic $\chi(X)$ is at most $1-(2 r-1)=2-2 r=\chi\left(S_{r}\right)$. Since no curve in $C$ is homotopically trivial, no component of $S_{r}-C$ is homeomorphic to a disc. Thus, no component of $S_{r}-C$ has positive Euler characteristic. It follows that $\chi(X)=\chi\left(S_{r}\right)$ and that every component of $S_{r}-C$ other than $X$ has an Euler characteristic of 0 (and that the polygon $S_{r}-\tau$ is simply connected).

Now let $\Sigma$ denote any collection of disjoint, smooth, simple closed curves on $S_{r}$, none of which is homotopically trivial, and no two of which are homotopic to one another. If $A$ is a subset of $\Sigma$ and $\gamma$ is a loop in $\Sigma-A$, then the surface $S_{r}-(A \cup\{\gamma\})$ either has one more component than $S_{r}-A$ or the total genus is one less than that of $S_{r}-A$. Thus, if we let $g$ denote the total genus of $S_{r}-\Sigma,|\Sigma|$ the number of loops in $\Sigma$, and $c$ the number of components of $S_{r}-\Sigma$, then

$$
g-c+|\Sigma|=r-1 .
$$

Hence, if $|\Sigma| \geq r+1$, then $g \leq c-2$, and there must be at least two components of $S_{r}-\Sigma$ that have genus 0 . Since $\Sigma$ consists of nontrivial, nonhomotopic loops, both of these two components must have negative Euler characteristics.

Therefore, if the multiple curve $C$ had more than $r$ nonhomotopic components, then there would be at least two components of $S_{r}-C$ which would have negative Euler characteristics.

\section{ACKNOWLEDGEMENTS}

The authors appreciate the contributions of anonymous referees, which included a generalization of the theorem and a simplification of the proof.

\section{REFERENCES}

1. Peter J. Cameron, Permutation groups, London Mathematical Society Student Texts, no. 45, Cambridge University Press, Cambridge, 1999. MR 2001c:20008

2. John D. Dixon and Brian Mortimer, Permutation groups, Graduate Texts in Mathematics, no. 163, Springer-Verlag, New York, 1996. MR 98m:20003 
3. Andrew Haas and Perry Susskind, The connectivity of multicurves determined by integral weight train tracks, Trans. Amer. Math. Soc. 329 (1992), no. 2, 637-652. MR 92e:57024

4. R. C. Penner and J. L. Harer, Combinatorics of train tracks, Annals of Math. Studies, no. 125, Princeton University Press, Princeton, NJ, 1992. MR 94b:57018

5. William Thurston, The geometry and topology of 3-Manifolds, Princeton University Press, Princeton, NJ, 1980.

Department of Mathematics, Southeastern Oklahoma State University, Durant, OKLAHOMA 74701

E-mail address: cmatthews@sosu.edu

Department of Mathematics, Oklahoma State University, Stillwater, Oklahoma 74075

E-mail address: wrightd@math.okstate.edu 